\title{
Prevalência do aleitamento materno exclusivo e fatores relacionados ao desmame precoce em um Hospital Amigo da Criança
}

Prevalence of exclusive breastfeeding and factors related to early weaning in a Child-Friendly Hospital

Prevalencia de la lactancia materna exclusiva y factores relacionados con el destete temprano en un Hospital Amigo de Niños

Larissa Arielly Cunha da Silva ORCID: https://orcid.org/0000-0002-1929-2272 Universidade Federal do Rio Grande do Norte, Brasil E-mail: larissarielly@ hotmail.com

Tatiana Lima da Silva Fernandes ORCID: https://orcid.org/0000-0002-8061-0989 Universidade do Estado de Mato Grosso, Brasil E-mail: mellima9@yahoo.es

Izabel Virginia Lima de Moura ORCID: https://orcid.org/0000-0001-6632-8680 Universidade Federal do Rio Grande do Norte, Brasil E-mail: iza.virginia@gmail.com Flávia de Araújo Gomes ORCID: https://orcid.org/0000-0001-8442-4633 Universidade Federal do Rio Grande do Norte, Brasil E-mail: flavia2001araujo@gmail.com

Débora de Almeida Aloise

ORCID: https://orcid.org/0000-0002-8678-4015 Universidade Federal do Rio Grande do Norte, Brasil E-mail: debora_aloise@yahoo.com.br

\begin{abstract}
Resumo
Objetivou-se estimar a prevalência do aleitamento materno exclusivo em crianças residentes no município de Santa Cruz - RN e identificar os fatores associados ao desmame precoce. Estudo longitudinal, de caráter quantitativo, realizado no período de abril/2019 a novembro/2020, teve como público alvo mães e suas respectivas crianças nascidas em um Hospital Amigo da Criança. Um total de 152 crianças/mães foram acompanhadas por meio de visitas mensais em domicílio. Para coleta de dados utilizou-se um questionário estruturado contendo variáveis relacionadas aos dados socioeconômicos, amamentação, gestação e parto. Os dados coletados foram organizados nos programas Epidata (versão 3.1) e Excel (versão 2010). Para a análise utilizou-se o programa STATA (versão 10.0). A introdução de outros leites começou no $1^{\circ}$ mês em $1,3 \%$ das crianças. Os principais motivos relatados pelas mães para o desmame precoce foram leite insuficiente $(16,6 \%)$ e rejeição por parte do bebê $(16,6 \%)$. No $6^{0}$ mês, a prevalência do AME foi de apenas $8,6 \%$, enquanto $60,5 \%$ realizavam o aleitamento materno complementado. O desmame precoce se associou à idade materna $(\mathrm{p}=0,035)$, ao tipo de parto $(\mathrm{p}=0,003)$, e a dificuldade de sucção do leite $(\mathrm{p}=0,022)$. Esse trabalho é pioneiro na região, os conhecimentos obtidos nesse estudo podem contribuir para a implantação de programas e adoção de medidas visando estimular a prática do AME.
\end{abstract}

Palavras-chave: Aleitamento materno; Estudos de prevalência; Desmame.

\begin{abstract}
The objective was to estimate the prevalence of exclusive breastfeeding in children living in the municipality of Santa Cruz - RN and to identify the factors associated with early weaning. A longitudinal study, of a quantitative character, carried out from April / 2019 to November / 2020, aimed at mothers and their respective children born in a BabyFriendly Hospital. A total of 152 children / mothers were followed up through monthly home visits. For data collection, a structured questionnaire was used containing variables related to socioeconomic data, breastfeeding, pregnancy and childbirth. The collected data were organized in the Epidata (version 3.1) and Excel (version 2010) programs. For the analysis, the STATA program (version 10.0) was used. The introduction of other milks started in the 1 st month in $1.3 \%$ of the children. The main reasons reported by mothers for early weaning were insufficient milk $(16.6 \%)$ and rejection by the baby $(16.6 \%)$. In the 6th month, the prevalence of EBF was only $8.6 \%$, while $60.5 \%$
\end{abstract}


performed complementary breastfeeding. Early weaning was associated with maternal age $(\mathrm{p}=0.035)$, type of delivery ( $\mathrm{p}=0.003)$, and difficulty in sucking milk $(\mathrm{p}=0.022)$. This work is pioneering in the region, the knowledge obtained in this study can contribute to the implementation of programs and the adoption of measures aimed at stimulating the practice of EB.

Keywords: Breast feeding; Cross-sectional studies; Weaning.

\section{Resumen}

El objetivo fue estimar la prevalencia de lactancia materna exclusiva en niños residentes en la ciudad de Santa Cruz RN e identificar los factores asociados al destete precoz. Estudio longitudinal, cuantitativo, realizado desde abril / 2019 hasta noviembre / 2020, tuvo como público objetivo a las madres y sus respectivos hijos nacidos en un Hospital Amigo del Niño. Un total de 152 niños / madres fueron seguidos a través de visitas domiciliarias mensuales. Para la recolección de datos utilizamos un cuestionario estructurado que contiene variables relacionadas con datos socioeconómicos, lactancia materna, embarazo y parto. Los datos recolectados se organizaron en los programas Epidata (versión 3.1) y Excel (versión 2010). Para el análisis se utilizó el programa STATA (versión 10.0). La introducción de otras leches se inició en el primer mes en el 1,3\% de los niños. Las principales razones informadas por las madres para el destete precoz fueron la insuficiencia de leche $(16,6 \%)$ y el rechazo del bebé $(16,6 \%)$. En el sexto mes, la prevalencia de LME fue solo del 8,6\%, mientras que el 60,5\% estaba recibiendo lactancia materna complementada. El destete precoz se asoció con la edad materna $(\mathrm{p}=0,035)$, el tipo de parto $(\mathrm{p}=0,003)$ y la dificultad para succionar la leche $(p=0,022)$. Este trabajo es pionero en la región, el conocimiento obtenido en este estudio puede contribuir a la implementación de programas y la adopción de medidas para incentivar la práctica de la LME.

Palabras clave: Lactancia materna; Estudios de prevalencia; Destete.

\section{Introdução}

O Aleitamento Materno (AM) além de ser uma estratégia natural de afeto, vínculo, proteção e nutrição para a criança, é também uma intervenção eficaz para a redução da morbimortalidade infantil. Amamentar é muito mais que nutrir, envolve interação entre mãe e filho, repercute no estado nutricional da criança e em sua habilidade de se defender de infecções, em sua fisiologia, desenvolvimento e na saúde a longo prazo. Assim, cabe ao profissional de saúde identificar e compreender o aleitamento materno no contexto sociocultural e familiar, e a partir daí dar suporte ativo às mães e à família (Brasil, 2015).

Observou-se o papel positivo da amamentação em bebês nascidos a termo, porém com o comprimento inferior para sua idade gestacional, onde o aleitamento materno pareceu impedir futuros problemas de saúde associados à obesidade e ao alto risco de doenças crônicas não transmissíveis nessas crianças. Quando amamentadas, essas crianças conseguiram recuperar seu crescimento sem prejuízo nutricional, reforçando a importância do aleitamento nos anos iniciais de vida (Santiago, et al., 2019).

Assim, além de suprir as necessidades do recém-nascido, a amamentação é uma importante estratégia preventiva de saúde pública no Brasil e no mundo. Diante disso, a Organização Mundial da Saúde (OMS) e o Ministério da Saúde (MS) indicam o Aleitamento Materno Exclusivo (AME) durante os seis primeiros meses de vida e posteriormente ser complementado até os dois anos de idade da criança (Brasil, 2015).

A ampliação das práticas de amamentação a níveis quase universais poderia prevenir 823.000 mortes infantis por ano em países de baixa e média renda (Moraes et al., 2016). A OMS quer garantir que até 2025, pelo menos metade de todas as crianças do mundo sejam amamentadas exclusivamente até os seis meses de idade (Oms, 2019).

Além de benefícios ao bebê, o aleitamento também traz benéficos à mãe, podendo protegê-la dos sintomas de depressão pós-parto. A autoeficácia da prática da amamentação é um fator protetor para o AME, e a cessação do AME apresenta uma associação estatística com sintomas de depressão pós-parto (Vieira et al., 2018).

Um estudo já realizado, mostrou que a prevalência tanto do AM como do AME são inversamente proporcionais aos meses de vida das crianças. Além disso, mostrou também que as diferentes regiões do país variam na prevalência do AM, sendo a região Norte a que apresenta os melhores índices (45,9\%), seguida das regiões Centro-Oeste (45\%), Sul (43,9\%), 
Sudeste $(39,4 \%)$ e Nordeste (37\%) e a prevalência nas áreas rurais ainda é maior que nas áreas urbanas (Pivetta et al., 2018). Dados do ENANI, 2019 mostram que a prevalência do AME entre as crianças menores de seis meses foi de 45,7\% no Brasil, sendo mais frequente na região Sul $(53,1 \%)$, e menos prevalente na região Nordeste $(38,0 \%)$.

De acordo com o IBGE, a taxa de mortalidade infantil média no município de Santa Cruz - RN é de 10,66 óbitos por 1.000 nascidos vivos. Comparado com todos os municípios do estado do Rio Grande do Norte, a cidade fica na posição $81^{\circ}$ de $167^{\circ}$ (Ibge, 2017).

Tendo em vista que diversos estudos apontam para um baixo índice de adesão ao aleitamento materno exclusivo em algumas regiões do Brasil, e entendendo a importância do AME, objetivou-se estimar a prevalência do aleitamento materno exclusivo até os seis primeiros meses de vida em um Hospital Amigo da Criança em crianças residentes no município de Santa Cruz - RN, além de identificar os motivos que levaram ao desmame precoce, uma vez que não existem dados descritos na literatura relacionados a esse tema na região.

\section{Metodologia}

Trata-se de um estudo do tipo longitudinal, com abordagem quantitativa, que segundo Mussi, et al. (2019), é baseado na análise dos componentes separadamente. De certa forma, essas informações, não são capazes de resolver os problemas, mas diante do rigor metodológico, permitem o acesso e a divulgação de informações valiosas. Realizado no município de Santa Cruz (RN), localizada à 114 km de Natal, capital do estado do Rio Grande do Norte e de acordo com as Unidades Básicas de Saúde a cidade se organiza em cinco áreas, sendo elas: Centro, Paraíso, Maracujá, DNER e Cônego Monte (Ibge, 2017).

O município conta com um Hospital Amigo da Criança que é referência na atenção à saúde materno-infantil na região do Trairi e adjacências. A instituição atende em sua estrutura assistencial leitos da obstetrícia clínica (alojamento conjunto), PPP (pré-parto, parto e puerpério), obstetrícia cirúrgica (alojamento conjunto), ginecologia cirúrgica, clínica médica, pediatria e Unidade de Terapia Intensiva Neonatal (Ebserh, 2020). De acordo com registros do próprio hospital, e dentre os partos realizados neste hospital por mês, tem-se que 25 partos ocorrem em mulheres que residem na cidade de Santa Cruz.

A coleta de dados ocorreu no período de abril de 2019 a novembro de 2020. O público alvo compreendeu crianças nascidas no hospital no período de abril de 2019 a dezembro de 2019 e suas respectivas mães que residiam na cidade de Santa Cruz. As puérperas que se encontravam no alojamento conjunto ou no PPP foram convidadas a participar da pesquisa após serem devidamente esclarecidas sobre o objetivo do estudo, importância de sua colaboração, riscos e benefícios da pesquisa. Aquelas que aceitaram participar, assinaram um Termo de Consentimento Livre e Esclarecido (TCLE) e informavam o nome completo, endereço e contato telefônico.

As informações epidemiológicas foram coletadas por meio de entrevistas previamente agendadas por telefone. O questionário desenhado para o estudo foi aplicado no primeiro momento de contato com as puérperas, e ocorria em local tranquilo e isolado, de forma a não gerar constrangimento diante das perguntas. As entrevistas com as mães ocorriam presencialmente no domicílio uma vez a cada quatro meses, iniciando no primeiro mês de vida da criança e finalizando no $12^{\circ}$ mês. Os meses que intercalaram as visitas domiciliares, as informações eram obtidas por meio do telefone, ou seja, ao todo foram realizadas quatro visitas presenciais e nos outros meses o contato se dava por telefone. As voluntárias respondiam a um questionário contendo questões sobre os aspectos sociodemográficos, prática do aleitamento materno, gestação e comportamento alimentar da mãe e do recém-nascido. Porém nem todas as mães puderam ter as quatro visitas domiciliares em virtude da pandemia do COVID19. A partir de março de 2020 as visitas domiciliares foram suspensas e os dados passaram a ser obtidos apenas por telefone. 
Todo o procedimento realizado nesse estudo foi aprovado pelo comitê de ética da Faculdade de Ciências da Saúde do Trairi e seguiu a Resolução no 466/2012 do Conselho Nacional de Saúde do Ministério da Saúde - Brasília - DF tendo seu início após aprovação pelo comitê de ética (parecer 5568 /CAAE 09027719.6.0000.5568).

Para se estabelecer o número mínimo amostral foi utilizado o programa OpenEpi (versão 3.03a). Para o cálculo considerou-se a prevalência média do aleitamento materno exclusivo no estado do Rio Grande do Norte de 20\%, média de 25 crianças nascidas por mês e que são residentes em Santa Cruz (totalizando uma população de 300 crianças por ano), intervalo de confiança de $95 \%$ e erro de 5\%. O resultado mostrou que o tamanho mínimo da amostra necessária para esse estudo era de 150 mães/crianças (binômio).

A amostragem ocorreu de forma aleatória, porém obedecendo aos critérios de inclusão onde a mãe e a criança deveriam residir em Santa Cruz - RN. As crianças que apresentavam alguma doença congênita foram excluídas da pesquisa.

Os riscos para as participantes da pesquisa foram mínimos. Na aplicação do questionário epidemiológico algumas questões poderiam particularmente causar algum constrangimento à mãe e a mesma poderia se recusar a responder qualquer pergunta sem prejuízo de participação na pesquisa. A aplicação do questionário foi realizada em local reservado para minimizar esses riscos.

Os benefícios da pesquisa foram estabelecidos com base no acompanhamento de crescimento e desenvolvimento das crianças, contribuindo para o estado de saúde das mesmas. As mães voluntárias recebiam informações acerca do aleitamento materno exclusivo até os seis meses de vida e foram estimuladas a continuar com essa prática até os dois anos ou mais da criança.

Para análise estatística foi utilizado o programa Epidata (versão 3.1) e um banco de dados foi gerado com base nas informações obtidas dos questionários. Para análise dos dados e cálculo do qui-quadrado, utilizou-se o programa STATA (versão 10.0). O teste de Qui-quadrado de Pearson permitiu comparar as frequências entre mães que realizaram o desmame precoce e aquelas que não o fizeram.

\section{Resultados}

Um total de 181 voluntárias participaram inicialmente da pesquisa, porém o acompanhamento completo por 12 meses foi realizado em 152 mães/crianças. Os motivos da perda de seguimento foram: mudança de domicílio, mudança de contato telefônico, troca de residência para outra cidade ou zona rural e desinteresse pelo projeto.

As participantes apresentavam idade entre 14 e 41 anos, sendo observada uma maior frequência (25\%) de mães com idade variando entre 20 e 24 anos. O grau de escolaridade foi distribuído em categorias onde se observou maior frequência $(32,2 \%)$ na categoria "Ensino médio completo". A profissão das voluntárias foi bem diversificada, destacando-se as ocupações de "dona de casa", "Agricultor" e "Profissional liberal". Em relação à renda familiar, a maioria das mães $(72,4 \%)$ relatou receber até 1 salário mínimo (Tabela 1). 
Características socioeconômicas e aspectos relacionados à gestação, parto e amamentação

Tabela 1. Características socioeconômicas das mães e crianças da pesquisa. Santa Cruz - RN, Brasil, 2020

\begin{tabular}{|c|c|c|}
\hline Características das voluntárias & $\mathrm{N}=152$ & $\%$ \\
\hline \multicolumn{3}{|l|}{ ASPECTOS SOCIOECONÔMICOS } \\
\hline \multicolumn{3}{|l|}{ Idade } \\
\hline Até 14 anos & 2 & 1,3 \\
\hline 15 a 19 anos & 15 & 9,8 \\
\hline 20 a 24 anos & 38 & 25,0 \\
\hline 25 a 29 anos & 29 & 19,1 \\
\hline 30 a 34 anos & 34 & 22,4 \\
\hline Acima de 35 anos & 34 & 22,4 \\
\hline \multicolumn{3}{|l|}{ Grau de Escolaridade } \\
\hline Ensino Fundamental incompleto & 30 & 19,7 \\
\hline Ensino Fundamental completo & 39 & 25,7 \\
\hline Ensino Médio incompleto & 13 & 8,6 \\
\hline Ensino Médio completo & 49 & 32,2 \\
\hline Ensino Superior (incompleto/completo) & 14 & 9,2 \\
\hline \multicolumn{3}{|l|}{ Renda Familiar } \\
\hline Até 1 salário mínimo & 110 & 72,4 \\
\hline De 1 a 2 salários mínimos & 21 & 13,8 \\
\hline De 2 a 3 salários mínimos & 6 & 3,9 \\
\hline De 3 a 4 salários mínimos & 2 & 1,3 \\
\hline De 4 salários mínimos ou mais & 8 & 5,3 \\
\hline Não sabe & 4 & 2,6 \\
\hline Não respondeu & 1 & 0,7 \\
\hline \multicolumn{3}{|l|}{ Bairro } \\
\hline Centro & 12 & 7,9 \\
\hline Maracujá & 39 & 25,7 \\
\hline Paraíso & 63 & 41,4 \\
\hline DNER & 14 & 9,2 \\
\hline Cônego Monte & 24 & 15,8 \\
\hline \multicolumn{3}{|l|}{ Profissão/Ocupação } \\
\hline Profissional liberal (Autônomo) & 26 & 17,1 \\
\hline Servidor público & 7 & 4,6 \\
\hline Empregado de empresa privada & 11 & 7,2 \\
\hline Empregado rural & 1 & 0,7 \\
\hline Agricultor & 33 & 21,7 \\
\hline Desempregado & 3 & 2,0 \\
\hline Do lar (dona de casa) & 58 & 38,2 \\
\hline Doméstica mensalista/diarista & 3 & 2,0 \\
\hline Estudante & 10 & 6,5 \\
\hline
\end{tabular}

Fonte: Autores.

O tempo de gestação foi distribuído em três categorias, prevalecendo o tempo de 37 a 42 semanas de gestação $(92,1 \%)$. O número de mães que tiveram parto normal $(\mathrm{N}=72)$ foi bem próximo ao número de mães que tiveram parto cesáreo (N=79). Com relação ao número de consultas de pré-natal, 58,5\% das mães relataram ter realizado 7 ou mais consultas durante a gestação, e 79,6\% das mães acompanhadas receberam orientações sobre o aleitamento materno no pré-natal (Tabela 2).

A maioria das mães $(96,7 \%)$ iniciaram o aleitamento ainda no hospital, e as que não iniciaram foi devido à baixa produção de leite $(1,3 \%)$, o bebê não conseguiu pegar o peito $(1,3 \%)$ e sorologia positiva para HIV $(0,7 \%)$. Das mães que 
conseguiram amamentar no hospital, 81,7\% iniciaram logo durante a $1^{\mathrm{a}}$ hora de vida do bebê (Tabela 2). Em relação ao tempo de amamentação, 51 mães pretendiam amamentar até os seis meses de vida da criança (Tabela 2), como é preconizado pela OMS. No entanto, dessas mães, 14 iniciaram o desmame precoce já no segundo mês (dados não mostrados), ofertando outros tipos de leite. Destaca-se ainda que no primeiro mês, 145 mães amamentavam, enquanto 7 já haviam deixado de amamentar logo após o $1^{\circ}$ mês. Ao final dos 12 meses, 91 mães relataram ainda amamentar, enquanto 61 haviam parado.

Os dois principais motivos do desmame precoce Ttabela 2) relatados pelas mães foram o leite insuficiente $(16,5 \%) \mathrm{e}$ rejeição por parte do bebê (16,5\%). Destaca-se ainda que 24,3\% das mães sentiram dificuldades em relação à pega ou sucção do leite, sendo a pega errada (16,4\%) o motivo mais prevalente. Observou-se ainda que $17,7 \%$ das mães acompanhadas iniciaram o desmame logo no primeiro mês de vida da criança (Tabela 2).

Tabela 2. Aspectos relacionados à gestação, parto e amamentação das mães e crianças da pesquisa. Santa Cruz - RN, Brasil, 2020.

\section{Características das voluntárias}

$\mathbf{N}=152$

\section{ASPECTOS RELACIONADOS À GESTAÇÃO E PARTO}

Tempo de gestação

Menos de 37 semanas/pré-termo

De 37 a 42 semanas/a termo

Acima de 42 semanas/pós-termo

Tipo de parto

Normal

Cesárea

Distórcia/Fórceps

Consultas de pré-natal realizadas

Nenhuma

1 a 3 consultas

4 a 6 consultas

7 ou mais consultas

Orientações sobre aleitamento no pré-natal

Sim

Não

Pretende amamentar por quanto tempo?

Menos de 6 meses

Até 6 meses

Acima de 6 meses

Dependendo do bebê

Enquanto produzir leite

Não sabe

Não se aplica

ASPECTOS RELACIONADOS AO ALEITAMENTO

MATERNO

Iniciou o aleitamento materno no hospital?

Sim

Não

Quando amamentou pela primeira vez?

$\begin{array}{cc}147 & 3,3\end{array}$

Durante a $1^{\mathrm{a}}$ hora de vida do bebê

Entre a $1^{\text {a }}$ e a $6^{\mathrm{a}}$ hora de vida

Após a $6^{\mathrm{a}}$ hora de vida

Quando parou de amamentar? 


$$
\begin{aligned}
& 3^{\circ} \text { mês } \\
& 4^{\circ} \text { mês } \\
& 5^{\circ} \text { mês } \\
& 6^{\circ} \text { mês } \\
& 7^{\circ} \text { mês } \\
& 8^{\circ} \text { mês } \\
& 9^{\circ} \text { mês } \\
& 10^{\circ} \text { mês } \\
& 11^{\circ} \text { mês } \\
& 12^{\circ} \text { mês } \\
& \text { Não se aplica }
\end{aligned}
$$

Motivos para o desmame precoce

Cirurgias anteriores na mama

Dor no peito, trauma emocional

Soropositiva para HIV

Leite insuficiente

Rejeição por parte do bebê

Retorno ao trabalho

Vontade própria da mãe

Não se aplica

A criança sentiu dificuldades com relação à "pega" ou sucção do leite?

Sim

Não

Dificuldades para sucção relacionava-se a:

Bebê gripado

Ingurgitamento mamário

Mamilo ferido

Mamilo ferido e pega errada

Mamilo invertido

Pega errada

Sucção não efetiva

Não se aplica

Quando introduziu outro tipo de leite, suco ou alimento?

$1^{\circ}$ mês
$2^{\circ}$ mês
$3^{\circ}$ mês
$4^{\circ}$ mês
$5^{\circ}$ mês
$6^{\circ}$ mês
$7^{\circ}$ mês
$8^{\circ}$ mês
$9^{\circ}$ mês
$10^{\circ}$ mês
$11^{\circ}$ mês
$12^{\circ}$ mês

Fonte: Autores.

\section{Prevalência dos tipos de Aleitamento Materno}

No $1^{\circ}$ mês, o AME foi predominante em 80,3\% $(n=122)$ das mães. No entanto, 4,61\% delas já realizavam o uso de fórmulas ou outros leites artificiais, 13,2\% estavam em AM misto ou parcial, e 2,0\% em AM predominante. No decorrer do acompanhamento, a porcentagem de mães que faziam o uso de fórmulas foi aumentando, e consequentemente a prática do AME foi diminuindo. No $6^{\circ}$ mês, apenas 8,6\% realizavam a prática do AME, enquanto 60,5\% estavam em aleitamento materno complementado. Apenas uma mãe continuou em $\operatorname{AME~}(0,7 \%)$ até o $8^{\circ}$ mês. Com 12 meses, a maioria estava em aleitamento complementado (59,2\%), 1 estava em aleitamento misto ou parcial, e 40,1\% já fazia uso de fórmulas (Figura 1). 
Figura 1. Prevalência dos tipos de aleitamento até o $6^{\circ}$ mês de vida das crianças e sua associação com o desmame precoce (valores de p). Santa Cruz, RN, Brasil, 2020.

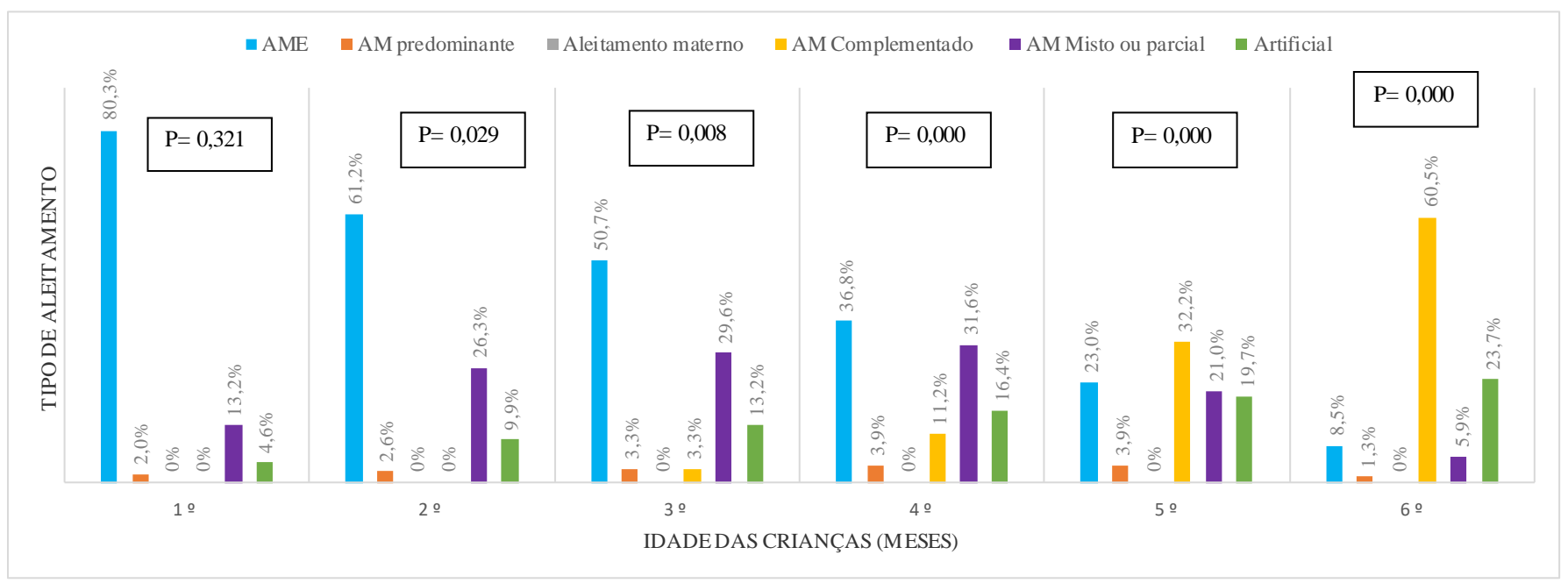

Fonte: Autores.

\section{Análise estatística (Teste Qui-quadrado de Pearson)}

Analisando as características socioeconômicas das mães, observou-se diferença significativa $(\mathrm{p}=0,035)$ entre as faixas de idade, onde $100 \%$ das mães com idade igual ou inferior a 24 anos realizaram o desmame precoce (Tabela 3).

Tabela 3. Relação entre as características socioeconômicas e a realização do desmame precoce por mães residentes no município de Santa Cruz - RN, Brasil, 2020.

\begin{tabular}{|c|c|c|c|}
\hline \multirow{2}{*}{$\begin{array}{l}\text { Características das voluntárias } \\
\qquad(\mathrm{N}=152)\end{array}$} & \multicolumn{2}{|c|}{ Desmame precoce } & \multirow{2}{*}{$\mathbf{P}$} \\
\hline & Não $(\%)$ & $\operatorname{Sim}(\%)$ & \\
\hline \multicolumn{4}{|l|}{ Idade (anos) } \\
\hline$\leq 14$ & $0(0,00)$ & $2(100)$ & \\
\hline$\geq 15$ e $\leq 19$ & $0(0,00)$ & $15(100)$ & \\
\hline$\geq 20$ e $\leq 24$ & $0(0,00)$ & $38(100)$ & 0,035 \\
\hline$\geq 25$ e $\leq 29$ & $2(6,90)$ & $27(93,10)$ & \\
\hline$\geq 30$ e $\leq 34$ & $7(20,59)$ & $27(79,41)$ & \\
\hline$\geq 35$ & $4(11,76)$ & $30(88,24)$ & \\
\hline \multicolumn{4}{|l|}{ Grau de Escolaridade } \\
\hline Analfabeta & $1(14,29)$ & $6(85,71)$ & \\
\hline Ensino Fundamental incompleto & $5(16,67)$ & $25(83,33)$ & \\
\hline Ensino Fundamental completo & $2(5,13)$ & $37(94,87)$ & 0,385 \\
\hline Ensino Médio incompleto & $1(7,69)$ & $12(92,31)$ & \\
\hline Ensino Médio completo & $2(4,08)$ & $47(95,92)$ & \\
\hline Ensino Superior (incompleto/completo) & $2(14,29)$ & $12(85,71)$ & \\
\hline \multicolumn{4}{|l|}{ Renda Familiar } \\
\hline Até 1 salário mínimo & $10(9,09)$ & $100(90,91)$ & \\
\hline De 1 a 2 salários mínimos & $1(4,76)$ & $20(95,24)$ & 0,253 \\
\hline De 2 a 3 salários mínimos & $0(0,00)$ & $6(100)$ & \\
\hline De 3 a 4 salários mínimos & $1(50,0)$ & $1(50,0)$ & \\
\hline De 4 salários mínimos ou mais & $1(12,50)$ & $7(87,50)$ & \\
\hline
\end{tabular}




\begin{tabular}{|c|c|c|c|}
\hline \multicolumn{4}{|l|}{ Bairro } \\
\hline Centro & $1(8,33)$ & $11(91,67)$ & \\
\hline Maracujá & $1(4,17)$ & $23(95,83)$ & 0,682 \\
\hline Paraíso & $2(14,29)$ & $12(85,71)$ & \\
\hline DNER & $2(5,13)$ & $37(94,87)$ & \\
\hline Cônego Monte & $7(11,11)$ & $56(88,89)$ & \\
\hline \multicolumn{4}{|l|}{ Profissão/Ocupação } \\
\hline Profissional liberal (Autônomo) & $2(7,69)$ & $24(92,31)$ & \\
\hline Servidor público & $0(0,00)$ & $7(100)$ & \\
\hline Empregado de empresa privada & $2(18,18)$ & $9(81,82)$ & \\
\hline Empregado rural & $0(0,00)$ & $1(100)$ & 0,784 \\
\hline Agricultor & $2(6,06)$ & $31(93,94)$ & \\
\hline Desempregado & $0(0,00)$ & $3(100)$ & \\
\hline Do lar (dona de casa) & $7(12,07)$ & $51(87,93)$ & \\
\hline Doméstica mensalista/diarista & $0(0,00)$ & $3(100)$ & \\
\hline Estudante & $0(0,00)$ & $10(100)$ & \\
\hline
\end{tabular}

Fonte: Autores.

Em relação às características do parto, observou-se diferença significativa $(\mathrm{p}=0,003)$ entre os tipos de parto, onde 98,73\% das mães que tiveram parto cesáreo realizaram o desmame precoce (tabela 4). Além dos motivos relatados para o desmame precoce (como insuficiência de leite ou rejeição por parte do bebê), observou-se que o mamilo ferido e a "pega errada" estão associados ao desmame precoce $(\mathrm{p}=0,022)$ (Tabela 5).

Tabela 4. Relação entre as características da gestação e do parto com o desmame precoce em mulheres residentes no município de Santa Cruz - RN, Brasil, 2020.

\begin{tabular}{|c|c|c|c|}
\hline \multirow[t]{2}{*}{ Características da gestação e parto $(\mathrm{N}=152)$} & \multicolumn{2}{|c|}{ Desmame precoce } & \multirow{2}{*}{$\mathbf{P}$} \\
\hline & Não (\%) & $\operatorname{Sim}(\%)$ & \\
\hline \multicolumn{4}{|l|}{ Tempo de gestação } \\
\hline Menos de 37 semanas/pré-termo & $0(0,00)$ & $9(100)$ & \\
\hline De 37 a 42 semanas/a termo & $13(9,29)$ & $127(90,71)$ & 0,544 \\
\hline Acima de 42 semanas/pós-termo & $0(0,00)$ & $3(100)$ & \\
\hline \multicolumn{4}{|l|}{ Tipo de parto } \\
\hline Normal & $12(16,90)$ & $59(83,10)$ & \\
\hline Cesárea & $1(1,27)$ & $78(98,73)$ & 0,003 \\
\hline Distorcia/Fórceps & $0(0,00)$ & $1(100)$ & \\
\hline \multicolumn{4}{|l|}{ Consultas de pré-natal realizadas } \\
\hline Nenhuma & $0(0,00)$ & $1(100)$ & \\
\hline 1 a 3 consultas & $1(8,33)$ & $11(91,67)$ & 0,987 \\
\hline 4 a 6 consultas & $4(8,00)$ & $46(92,00)$ & \\
\hline 7 ou mais consultas & $8(8,99)$ & $81(91,01)$ & \\
\hline \multicolumn{4}{|l|}{ Orientações sobre Aleitamento no pré-natal } \\
\hline Não & $2(6,45)$ & $29(93,55)$ & 0,639 \\
\hline Sim & $11(9,09)$ & $110(90,91)$ & \\
\hline \multicolumn{4}{|l|}{ Pretende amamentar por quanto tempo? } \\
\hline Menos de 6 meses & $0(0,00)$ & $5(100)$ & \\
\hline Até 6 meses & $5(9,80)$ & $46(90,20)$ & \\
\hline Mais de 6 meses & $1(7,69)$ & $12(92,31)$ & 0,270 \\
\hline Enquanto produzir leite & $1(2,94)$ & $33(97,06)$ & \\
\hline Enquanto o bebê quiser & $3(9,68)$ & $28(90,32)$ & \\
\hline Não sabe & $3(27,27)$ & $8(72,73)$ & \\
\hline Não se aplica & $0(0,00)$ & $7(100)$ & \\
\hline
\end{tabular}


Tabela 5. Relação das características do aleitamento e o desmame precoce em crianças acompanhadas durante os 12 primeiros meses de vida. Santa cruz, RN, brasil, 2020.

\begin{tabular}{lccc} 
& \multicolumn{2}{c}{ Desmame precoce } & P \\
& Não (\%) & Sim (\%) \\
\hline Iniciou o aleitamento materno no hospiticas do aleitamento & & & \\
Sim & $13(8,84)$ & $134(91,16)$ & 0,487 \\
Não & $0(0,00)$ & $5(100)$
\end{tabular}

Quando amamentou pela primeira vez?

Durante a $1^{\mathrm{a}}$ hora de vida do bebê

Entre a $1^{\mathrm{a}}$ e a $6^{\mathrm{a}}$ hora de vida

Após a $6^{\mathrm{a}}$ hora de vida

Quando parou de amamentar?

$1^{\circ}$ mês
$2^{\circ}$ mês
$3^{\circ}$ mês
$4^{\circ}$ mês
$5^{\circ}$ mês
$6^{\circ}$ mês
$7^{\circ}$ mês
$8^{\circ}$ mês
$9^{\circ}$ mês
$10^{\circ}$ mês
$12^{\circ}$ mês

Motivos para o desmame precoce Cirurgias anteriores na mama

Dor no peito, trauma emocional

Soropositiva para HIV

Leite insuficiente

Rejeição por parte do bebê

Retorno ao trabalho

Vontade própria da mãe

A criança sentiu dificuldades com relação à "pega" ou sucção do leite?

$\operatorname{Sim}$

Não

\section{Dificuldades para sucção relacionava-se a:}

Bebê gripado

Ingurgitamento mamário

Mamilo ferido

Mamilo ferido e pega errada

Mamilo invertido

Pega errada

$\begin{array}{rr}5(13,51) & 32(86,49) \\ 8(6,96) & 107(93,04)\end{array}$

$\begin{array}{ccc}11(9,09) & 110(90,91) & \\ 2(11,76) & 15(88,24) & \\ 0(0,00) & 9(100) & 0,588\end{array}$

$\begin{array}{ll}0(0,00) & 4(100) \\ 0(0,00) & 7(100) \\ 0(0,00) & 5(100) \\ 0(0,00) & 5(100) \\ 0(0,00) & 5(100) \\ 0(0,00) & 6(100) \\ 0(0,00) & 5(100) \\ 2(20,00) & 8(100) \\ 0(0,00) & 6(100) \\ 0(0,00) & 2(100) \\ 0(0,00) & 2(100)\end{array}$

$\begin{array}{lr}0(0,00) & 1(100) \\ 0(0,00) & 1(100) \\ 0(0,00) & 1(100) \\ 0(0,00) & 25(100) \\ 1(4,00) & 24(96,00) \\ 1(25,00) & 3(75,00) \\ 0(0,00) & 3(100)\end{array}$

0,327

$\begin{array}{lr}1(100) & 0(0,00) \\ 1(100) & 0(0,00) \\ 0(0,00) & 4(100) \\ 0(0,00) & 1(100) \\ 0(0,00) & 1(100) \\ 2(8,00) & 23(92,00)\end{array}$

0,215

0,022 
Sucção não efetiva

Durante o AME, você ingeriu alimentos como chocolates, café, refrigerantes?

$\operatorname{Sim}$

Não
$1(25,00)$

$8(8,16)$
$3(75,00)$

$49(90,74)$

$90(91,84)$

Fonte: Autores.

\section{Discussão}

O processo de amamentação depende de diversos fatores associados, dentre eles, o momento em que a mulher está vivendo, condições de vida e de trabalho, das suas experiências anteriores, e da compreensão que a própria sociedade tem a respeito. Assim, a presença de fatores socioeconômicos e culturais interfere no processo de amamentação (Oliveira, et al., 2017).

Em nosso estudo, a maioria das mães tinham o ensino médio completo, renda familiar de até um salário mínimo e eram donas de casa, porém essas características não mostraram associação com o desmame precoce. Tal associação foi encontrada em um estudo realizado por Vieira e colaboradores (2019), onde 29,0\% das puérperas possuíam ensino médio completo, e 62,3\% tinham renda familiar de até um salário, além de 49,5\% delas serem solteiras. Os dados mostraram que $53,7 \%$ das mães tinham até 25 anos, e que esse grupo de mulheres jovens tendem a amamentar menos tempo, e na maioria das vezes esse comportamento está associado ao baixo nível de escolaridade, baixa renda e não serem casadas. Assim, esse grupo de mulheres foi caracterizado como grupo de risco para o desmame precoce (Vieira, et al., 2019).

Em nosso estudo, apesar de não encontrar associação entre o nível de escolaridade e a baixa renda com o desmame precoce, foi encontrado associação com a idade das mães ( $\mathrm{p}=0,035)$, onde todas as mães jovens com idade igual ou inferior a 24 anos realizaram o desmame precoce. Neri e colaboradores (2019) também encontraram associação entre o desmame precoce e idade da mãe. Esse estudo analisou mães de crianças de 0 a 12 meses residentes no Distrito Federal e no estado de Goiás. Os dados mostram que a idade materna com a maior prevalência de desmame precoce foi entre 20 e 30 anos (57,7\%). Essa faixa etária é a mais adequada para gestação, porém, traz ao mesmo tempo maiores dificuldades para as mães.

Um estudo realizado em Aracajú, capital do estado de Sergipe no nordeste brasileiro, mostrou que mulheres que recebem até um salário mínimo, que são predominantemente pardas e de baixa escolaridade apresentaram maior desigualdade de acesso a consultas no pré-natal, além de exames ginecológicos e laboratoriais, sendo um dos motivos para incentivar a implantação de programas de melhoria as consultas de pré-natal, as quais são oferecidas pelo SUS (Carvalho et al., 2016). Estudo caso-controle realizado em João Pessoa (PB) procurou identificar os fatores que se relacionavam a duração do aleitamento materno. A introdução precoce de fórmula infantil, de outros leites, e realizar menos de seis consultas de pré-natal foram fatores de risco para uma menor duração do aleitamento materno (Mendes, et al., 2019).

Em nosso estudo, 79,6\% $(\mathrm{n}=121)$ das mães relataram terem recebido orientações durante as consultas de pré-natal sobre o aleitamento materno, e destas mães apenas 11 permaneceram em AME. Tais dados mostram a necessidade de um programa mais efetivo na assistência ao pré-natal. O uso de protocolos durante essa assistência, visa reforçar a prestação de serviço e uma escuta qualificada através de orientações e educação em saúde, cadastramento da gestante em programas da atenção básica, fornecimento e preenchimento do cartão da gestante, consultas, solicitação de exames, prescrições, visitas domiciliares, acompanhamento e aconselhamento no aleitamento e planejamento familiar. Todas essas ações tem o intuito de tornar a assistência e consultas mais qualificadas (Oliveira, et al., 2016). 
Neri e colaboradores (2019) encontraram uma frequência maior $(95,3 \%)$ de mães que receberam orientações por profissionais de saúde sobre o aleitamento materno exclusivo, observando-se uma diferença significativa $(\mathrm{p}<0,01)$ quando comparado com mães que não receberam orientações $(4,7 \%)$. A falta de informações durante o pré-natal é uma das principais causas da ausência da amamentação visto que o pouco conhecimento faz uma mãe inexperiente acreditar em mitos sobre o aleitamento (Tenório et al., 2018). Algumas causas culturais/educacionais relacionadas ao desmame precoce em prematuros foram leite insuficiente/secou, crença no benefício do chá e necessidade de água (Lima et al., 2019). Em nosso estudo, todas as mães que tiveram parto prematuro ( $<37$ semanas) realizaram o desmame precoce e os motivos alegados foram leite insuficiente e rejeição por parte da criança.

Com relação ao tipo de parto, estudos mostram que a cesariana é um dos fatores de risco associados ao início tardio da amamentação, podendo até reduzir sua prevalência na primeira hora pós-parto devido a situações que retardam o contato entre mãe e filho, contribuindo para o desmame precoce (Vieira, et al., 2019). Em nosso estudo, observou-se associação entre o tipo de parto e desmame precoce $(\mathrm{p}=0,003)$. Das 79 mães que tiveram parto cesáreo, 74,7\% conseguiram amamentar durante a primeira hora de vida do bebê, porém apenas 1 dessas mães manteve o aleitamento materno exclusivo por 6 meses e continuou de forma complementada. Os motivos relatados por essas mães para o desmame precoce foram leite insuficiente, rejeição por parte do bebê, vontade própria da mãe e dor no peito.

$\mathrm{O}$ aparecimento de intercorrências nas mamas deve ser constantemente observado e avaliado, devendo ser tratado de forma correta pelos profissionais de saúde ainda na maternidade. Considera-se um achado importante, pois pode representar determinantes que dificultam a prática do aleitamento materno, podendo estar relacionados a alterações anatômicas nas mamas, posicionamento inadequado da mãe ou criança, controle inadequado da suçãao, entre outros fatores (Tenório et al., 2018). Em nosso trabalho, 37 mães relataram que seus filhos apresentaram dificuldade para a "pega" e sucção do leite, porém esse fator não mostrou associação com o desmame precoce. Entretanto, os motivos que levaram a essa dificuldade mostraram associação $(\mathrm{p}=0,022)$, indicando que a "pega errada" e mamilos feridos são fatores que reduzem a prevalência do AME.

Vieira e colaboradores (2015), também encontraram resultados semelhantes ao analisar a mamada de recém-nascidos nas primeiras 24 horas pós-parto. $\mathrm{O}$ aspecto relacionado à pega do bebê mostrou índice desfavorável, com escore ruim de 10\% da mãe e do bebê. A má pega torna-se um dos principais fatores causador de fissuras/traumas, ocasionando dor e contribuindo para a ineficácia da amamentação.

Amaral e colaboradores (2015) identificaram fatores que influenciavam as nutrizes na interrupção do AME durante os primeiros seis meses de vida do lactente. Este trabalho mostrou que a crença na produção insuficiente de leite, o aleitamento materno predominante, dificuldades de pega da mama, intercorrências com o neonato, e intercorrências mamárias são alguns desses fatores. Dentre essas intercorrências mamárias, as mais prevalentes foram o mamilo ferido e a dor. Muitos desses fatores se relacionam ao desconhecimento dos aspectos fisiológicos da lactação e preparo para essa nova fase da mãe, sendo necessário capacitar essas mulheres em suas decisões e manter um diálogo constante durante a gestação com profissionais da saúde.

Estudo realizado em Minas Gerais procurou avaliar a frequência do aleitamento materno e a introdução da alimentação complementar em crianças de 0 a 24 meses. No período de AME, as crianças já recebiam água e leite não materno, e a introdução complementar de guloseimas ocorreu precocemente. Percebe-se que a complementação do leite materno com líquidos não nutritivos, como água e chás, não é recomendada antes dos seis meses de idade, podendo contribuir para uma interrupção do AME (Lopes, et al., 2018).

Em nosso estudo observou-se uma queda nos resultados da prevalência do AME com o decorrer do tempo. No $1^{\circ}$ mês $80,3 \%$ das mães amamentavam exclusivamente, porém no $6^{\circ}$ mês essa frequência reduziu para $8,5 \%$. À medida que a 
frequência do AME reduzia, a do aleitamento do tipo artificial aumentava. No $1^{\circ}$ mês, $4,6 \%$ das mães usavam a fórmula para alimentar seus filhos, e no $6^{\circ}$ mês essa porcentagem passou para 23,7\%. A análise dos tipos de aleitamento mostrou associação entre a introdução de outros nutrientes e o desmame precoce, indicando diferença estatística já no segundo mês ( $\mathrm{p}=0,029)$.

O Ministério da Saúde recomenda a introdução de alimentos a partir dos seis meses (Brasil, 2015). O estudo de Lopes e colaboradores (2018) mostrou que a introdução complementar de alimentos como líquidos, mel, açúcar, e guloseimas se mostrou precoce, afetando diretamente o aleitamento materno de crianças envolvidas no estudo entre 0 e 24 meses. A oferta de alimentos antes desse período ocasiona prejuízos à saúde infantil.

Mesmo diante de diversas campanhas, ainda se observa no Brasil uma baixa adesão ao aleitamento materno. Sabe-se que a oferta de informações ainda é o principal meio para a resolução de diversos temas na área da saúde pública e que tais informações provenientes dessas ações trazem benefícios, tanto no âmbito hospitalar como familiar (Rocha, et al. 2018).

Com base na análise da tendência do aleitamento materno no Brasil, a prevalência do AME em menores de seis meses chegou a aumentar um total de 42,8 pontos percentuais entre os anos de 1986 e 2020, passando de 2,9\% para 45,7\% nesses 34 anos (Enani, 2019). Assim, são a partir de inovações nas unidades de serviço, apoio e acompanhamento da mãe e filho, que resultados positivos poderão surgir a favor do prolongamento do aleitamento materno (Mendes, et al., 2019).

\section{Conclusão}

Identificou-se que a maioria das mães nesse estudo tinham ensino médio completo, renda familiar de até um salário mínimo, e eram donas de casa. Apesar de outros estudos apontarem essas características como fator de risco para o desmame, não observamos tal associação. Contudo, a idade materna igual ou inferior a 24 anos mostrou associação com o desmame precoce.

A prevalência do AME no primeiro mês foi de $80,3 \%$, com redução para $8,5 \%$ no $6^{\circ}$ mês. $\mathrm{O}$ aleitamento materno complementado e o artificial tiveram um aumento no quarto e quinto mês, aumentando sua adesão com o passar dos meses. A introdução de alimentos do tipo líquido se deu ainda no primeiro mês em 1,3\% das crianças e a ingestão de alimentos do tipo sólidos foi observado no terceiro mês em $0,7 \%$ das crianças. Outros dois fatores relacionados ao desmame precoce foram o tipo de parto e a pega errada/mamilo ferido durante a sucção do bebê.

Destarte, o conhecimento obtido acerca da realização da pesquisa apresenta estimada relevância no sentido de propor aos profissionais da saúde um conhecimento sobre o perfil da prática do aleitamento materno exclusivo nas mães residentes em Santa Cruz-RN e permitem que trabalhem com essa abordagem nos diversos campos da atenção primária à saúde. Faz-se necessário o acompanhamento e orientações durante o período gestacional em vista da promoção e proteção à adesão ao aleitamento.

É de suma importância trabalhos como esse, visto a necessidade de posteriormente analisar a qualidade de vida e o desenvolvimento das mães e das crianças que passaram ou não pelo processo de aleitamento materno. Assim, isso se torna útil para mais ações voltadas ao tema principalmente no âmbito da atenção primária à saúde, como sugestões para trabalhos futuros.

\section{Referências}

Amaral, L. J. X. Sales, S. S. Carvalho, D. P. S. R. P., Cruz, G. K. P., Azevedo, I. C., \& Ferreira Júnior, M. A. (2015). Fatores que influenciam na interrupção do aleitamento materno exclusivo em nutrizes. Revista Gaúcha Enfermagem. 36(esp):127-34.

Brasil. (2020). Governo do Brasil. Ministério da educação. "HUAB: Nossa história”. 〈http://www2.ebserh.gov.br/web/huab-ufrn/nossa-historia>

Brasil. (2017). Instituto brasileiro de geografia e estatística. Governo do Brasil. Cidades e estados. <https://www.ibge.gov.br/cidades-eestados/rn/santacruz.html>. 
Research, Society and Development, v. 10, n. 9, e52510918375, 2021

(CC BY 4.0) | ISSN 2525-3409 | DOI: http://dx.doi.org/10.33448/rsd-v10i9.18375

Brasil. (2015). Ministério da Saúde. Secretaria de Atenção à Saúde. Departamento de Atenção Básica. Saúde da criança: aleitamento materno e alimentação complementar. Caderno de atenção básica n 23. (2a ed.).

Brasil. (2012). Ministério da Saúde. Resolução nº 466/2012 do Conselho Nacional de Saúde do Ministério da Saúde.

Carvalho, R. A. S. Santos,V. S. Melo, C. M., Gurgel, R. Q., \& Oliveira, C. C. C. (2016). Avaliação da adequação do cuidado pré-natal segundo a renda familiar em Aracajú, 2011. Epidemiologia e serviços de saúde. 25(2).

Lima, A. P. E, Castral, T. C., Leal, L. P., Javorski, M., Sette, G. C. S., Scochi, C. G. S., \& Vasconcelos, M. G. L. (2019). Aleitamento materno exclusivo de prematuros e motivos para sua interrupção no primeiro mês pós-alta hospitalar. Revista gaúcha Enfermagem. 40:e20180406.

Lopes, W. C.. Marques, F. K. S.. Oliveira, C. F.. Rodrigues, J. A., Silveira, M. F., Caldeira, A. P., \& Pinho, L. (2018). Alimentação de crianças nos primeiros dois anos de vida. Revista Paulista de Pediatria. 36(2):164-170.

Mendes, S. C. Lobo, I. K. V. Sousa, S. Q. \& Vianna, R. P. T. (2019). Fatores relacionados com uma menor duração total do aleitamento materno. Ciência \& Saúde Coletiva, 24(5):1821-1829.

Moraes, B. A., Gonçalves, A. C., Strada, J. K. R., \& Gouveia, H. G. (2016). Fatores associados à interrupção do aleitamento materno exclusivo em lactentes com até 30 dias. Revista Gaúcha de Enfermagem. 37(esp):e2016-0044.

Mussi, R. F. F., Mussi, L. M. P. T., Assunção, E. T. C., \& Nunes, C.P. (2019). Pesquisa quantitativa e/ou qualitativa: distanciamentos, aproximações e possibilidades. Revista SUSTINERE. 7, 414-430.

Neri, V. F.. Alves, A. L. L.. \& Guimarães, L. C. (2019). Prevalência de desmame precoce e fatores relacionados em crianças do Distrito Federal e entorno. REVISA. 8(4): 451-9.

Oliveira, C. M.. Santos, T. C.. Melo, I. M., \& Aguiar, D. T. (2017). Promoção do Aleitamento Materno: intervenção educativa no âmbito da Estratégia de Saúde da Família. Rev. Enfermagem Revista. V. 20, N.2.

Oliveira, E. C.. Barbosa, S. M.. \& Melo, S. E. P. (2016). A importância do acompanhamento pré-natal realizado por enfermeiros. Revista científica FacMais. Vol VII, número 3.

Organização Mundial da Saúde. (2019). "UNICEF: apenas 40\% das crianças no mundo recebem amamentação exclusiva no início da vida". Instituto Lado a Lado pela vida. <https://www.ladoaladopelavida.org.br/detalhenoticia-ser-informacao/unicef-apenas-40-das-criancas-no-mundo-recebemamamentacaoexclusiva-no-inicio-da-vida>.

Pivetta, H. M. F., Braz, M. M., Pozzebon, N. M., Freire, A. B., Real, A. A., Cocco, V. M., \& Sperandio, F. F. (2018). Prevalência de aleitamento materno e fatores associados: uma revisão de literatura. Revista de ciências médicas e biológicas. 17(1), 95-101.

Rocha, I. S. Lolli, L. F. Fujimaki, M., Gasparetto, A., \& Rocha, N. B. (2018). Influência da autoconfiança materna sobre o aleitamento materno exclusivo aos seis meses de idade: uma revisão sistemática. Ciência \& Saúde Coletiva. 23 (11):3609-3619.

Santiago, A. C. T, Cunha, L. P. M., Vieira, N. S. A., Moreira, L. M. O., Oliveira, P. R., Lyra, P. P. R., \& Alves, C. A. D. (2019). Breastfeeding in chil-dren born small for gestational age and future nutritional and metabolic outcomes: a systematic review. Jornal de Pediatria (Rio de Janeiro). 95:264---74.

Tenório, M. C. S. Mello, C. S. \& Oliveira, A. C. M. (2018). Factors associated with the lack of breastfeeding upon discharge from hospital in a public maternity facility in Maceió, Alagoas, Brazil. Ciência \& Saúde Coletiva, 23(11):3547-3556.

Ufrj. (2020). Universidade Federal do Rio de Janeiro. Estudo Nacional de Alimentação e Nutrição Infantil - ENANI-2019: Resultados preliminares Indicadores de aleitamento materno no Brasil. 9p. UFRJ.

Vieira, A. C. Costa, A. R. \& Gomes, P. G. (2015). Boas práticas em aleitamento materno: aplicação do formulário de observação e avaliação da mamada. Rev. Soc. Bras. Enf. Ped. 15(1), 13-20.

Vieira, E. S., Caldeira, N. T., Eugênio, D. S., Di Lucca, M. M., \& Silva, I. A. (2018). Breastfeeding self-efficacy and postpartum depression: a cohort study. Rev. Latino-Am. Enfermagem. 26:e3035.

Vieira, F. S.. Costa, E. S.. Sousa, G. C.. Oliveira, T. M. P., \& Neiva, M. J. L. M. (2019). Influência do parto sobre o desmame no puerpério. Rev Fund Care Online. 11, 425-431. 